Revista de Psicología Vol. 30 (2), 2012 (ISSN 0254-9247)

\title{
El Infant Facial Expressions of Emotions from Looking at Pictures. Versión peruana
}

\author{
Pierina Traverso ${ }^{1}$ y Magaly Nóblega ${ }^{2}$ \\ PUCP, Perú
}

Se presenta la versión peruana del Infant Facial Expressions of Emotions from Looking at Pictures (IFEEL), que evalúa la interpretación de emociones a partir de fotografías de rostros de nińos. La versión original del instrumento de Emde, Osofsky \& Butterfield (1993) fue construida en Estados Unidos y tiene 30 estímulos. La versión peruana considera 25 fotografías de niños con rasgos faciales comunes en la población peruana. A partir de las respuestas obtenidas con una muestra de 363 hombres y mujeres de nivel socioeconómico medio y bajo (19-45 años) se creó un léxico propio de 14 emociones que incluye las formas de verbalización para cada uno de los grupos de emoción obtenidos en la muestra peruana. La mayoría de los grupos muestra confiabilidad por estabilidad temporal. Finalmente, se halló que el nivel socioeconómico (NSE) es una variable que genera diferencias significativas en la manera como las personas interpretan las emociones y se crearon valores referentes de interpretación diferenciados a partir de esta variable. Palabras clave: disponibilidad emocional, evaluación de la interpretación de emociones, IFEEL

\section{The Infant Facial Expressions Of Emotions From Looking at Pictures. Peruvian version}

The Peruvian version of the Infant Facial Expression of Emotions from Looking at Pictures (IFEEL), instrument that assessed the interpretation of emotions from children's faces pictures is presented. The original version from Emde, Osofsky \& Butterfield (1993) was developed in the United States and involves 30 stimuli. The Peruvian version involves 25 pictures of children with prototypic facial features of the majority of Peruvian population. A sample of 363 men and women of middle and low socio-economic status between 19 and 45 years old was recruited to develop the Peruvian version. From the results, a lexicon was created with the words that were used by the participants to designate the 14 groups of emotion that were obtained. The majority of these groups had an adequate reliability for temporal stability. Finally, it was found that the socio-economic status (SES) is a variable that generates significant differences in the way how persons interpret the emotions. Therefore, referential values of differentiated interpretation were created from this variable.

Key words: emotional availability, interpretation of emotions, IFEEL

1 Doctora en Psicología. Docente del Departamento de Psicología de la PUCP. Contacto: Av. Las Acacias 196-601, Lima 18-Perú, ptraver@pucp.edu.pe

2 Magíster en Salud Mental en Poblaciones. Docente del Departamento de Psicología de la PUCP. Contacto: Av. Ramírez Gastón 266-302, Lima 18-Perú; mnoblega@pucp.edu.pe 

A lo largo de los años, diversos autores (Emde, 1993; Fonagy, Gergely, Jurist \& Target, 2002; Stern, 1991; Winnicott, 1982) han resaltado el vínculo entre un bebé y su madre como aquella relación temprana que tiene mayor trascendencia en el desarrollo de la vida psíquica del infante. Así, una interacción continua y consistente entre el recién nacido y su madre $-\mathrm{o}$ aquella persona encargada de cumplir ese rol- constituye la condición esencial para que, progresivamente, el bebé adquiera una experiencia de sí mismo como un organismo con una mente, capaz de autorregularse (Brazelton \& Cramer, 1993). En otras palabras, el vínculo del infante con su madre es, en gran parte, aquel que permite que un bebé desarrolle la capacidad de controlar y modular sus respuestas fisiológicas y afectivas, transformándolas en pensamiento. Esto a su vez permitirá, con el tiempo, que el bebé logre obtener una comprensión de sí mismo y, a partir de ella, de las personas que lo rodean.

En esta interacción, las expresiones emocionales se convierten en el "lenguaje común" entre la madre y el recién nacido, ya que a través de ellas el bebé expresa sus necesidades y su estado de ánimo, permitiéndole a la madre acceder al mundo interno de su bebé y responder a éste. Para ello, más allá de una madre físicamente presente, se requiere de una madre que esté psicológica y afectivamente disponible, de modo que pueda percibir, entender y responder adecuadamente a las señales emocionales que el infante va emitiendo. Así, si bien el bebé tiene un rol activo en su propio desarrollo y en la interacción con su madre, la responsabilidad sobre su desarrollo psíquico recae casi completamente sobre ella; específicamente, sobre su disposición y sensibilidad para conectarse con su bebé y adaptarse a él (Emde, 1993; Fonagy et al., 2002). 
Un aspecto central de un ambiente cuidador adaptativo es la disponibilidad emocional (Emde, 1980), entendida como un constructo diádico que alude a los intercambios que regulan la comunicación emocional entre el infante y su cuidador. En la disponibilidad emocional, entonces, está implicada la calidad de los intercambios afectivos, así como la capacidad emocional de la madre para percibir y responder a las motivaciones y señales emocionales que emite su bebé (Emde, 1980). A la base de esta disponibilidad se encuentra la mentalización (Fonagy \& Target, 1997), considerada como un aspecto central del funcionamiento humano ya que permite reconocerse a uno mismo y a los demás como seres pensantes e intencionales. A través de esta capacidad, la madre podrá percibir al bebé como una entidad psicológica independiente, entendiendo que sus expresiones emocionales tienen la intención de comunicar algo y así responder a sus necesidades a partir de esta percepción. El hecho que la madre refleje la mente y las emociones del bebé en sus propios pensamientos, constituye la base para que este logre una comprensión de sí mismo y así desarrolle la capacidad para mentalizar, permitiéndole luego, establecer intercambios vinculares en términos mentales. De este modo, el desarrollo de la capacidad de mentalización de un bebé depende de la capacidad de mentalización de su madre (Emde, 1993; Schejtman, 2008).

Otro aspecto esencial de la disponibilidad emocional de una madre es el affective mirroring (Fonagy et al., 2002). Diversos autores señalan que la capacidad de la madre para actuar como "espejo" de los estados afectivos del infante es esencial para su desarrollo, ya que le permite a éste percibir sus afectos como algo reconocible y compartido con otro. Además, le permite aprender, progresivamente, a transformar y regular sus cambiantes estados afectivos, internalizando la función reguladora de afecto materno (Fonagy et al., 2002). Este concepto de funcionamiento en espejo es similar al concepto de Winnicott (1982), quien señala que el niño puede conocerse a sí mismo a través de la mirada de su madre, favoreciendo el desarrollo de un sentido de self. Por lo anterior, entendemos que el procesamiento que la madre hace de la información emocional del infante es de importancia crucial para el desarrollo 
psíquico de este. Más aún, la interpretación de emociones, definida como el significado subjetivo que una persona otorga a la experiencia afectiva de un otro (Ackerman \& Izard, 2004), se convierte en un constructo importante a estudiar, ya que el grado de sensibilidad que tenga la madre para otorgar significados adecuados a las expresiones emocionales de su bebé tiene una influencia directa en su desarrollo psíquico.

Así, la adecuada interpretación de las emociones del niño por parte de su madre permitirá sentar una base para su vida emocional posterior, al permitirle reconocer y procesar sus propias emociones, dándole forma y sentido a su experiencia (Brazelton \& Cramer, 1993; Emde, 1993; Meurs \& Vliegen, 2009; Winnicott, 1962). Las madres no solo reaccionan a la naturaleza objetiva de esta conducta, sino también a la interpretación que hacen de ella, la cual se encuentra influida por su mundo subjetivo (Brazelton \& Cramer, 1993). Por tal motivo, es importante tener en cuenta no solo la personalidad e historia temprana de vida de las madres, sino también ciertas variables culturales o contextuales, las cuales imprimirán un sello particular en su modo de comprender las emociones de sus bebés.

Cuando una madre interpreta erróneamente las expresiones corporales-afectivas del niño de manera sistemática, genera en él una dificultad para etiquetar apropiadamente sus estados internos, los cuales permanecerán confusos, no simbolizados y difíciles de ser regulados (Fonagy et al., 2002). Entendemos entonces que una interpretación sensible de las emociones del infante es otro componente esencial de la disponibilidad emocional, y su ausencia socava un importante pilar del desarrollo de la regulación afectiva, generándose un riesgo para la aparición de respuestas conductuales maladaptativas, así como para el desarrollo de la psicopatología (Emde, 1993). Así, por ejemplo, se ha encontrado que los afectos, la regulación afectiva y la posterior seguridad en el vínculo de niños con madres depresivas, guardan relación con el afecto y la conducta de sus madres, la cual demuestra un menor número de interacciones afectivas contingentes, mayor intrusividad y mayor expresión de afecto negativo (Field, 1994; Field et al., 1988; Murray, 1992 entre otros citados en Fonagy et al., 2002). 
Si bien es clara la necesidad de evaluar el modo cómo los cuidadores interpretan las señales emocionales de los infantes, los instrumentos diseñados para este propósito han sido escasos, y en el Perú no se cuenta con ninguno. Es así que surge la necesidad de tener algún instrumento que nos permita evaluar el constructo de "interpretación de emociones" adaptado para nuestro contexto, con el objetivo de detectar poblaciones en la cuales haya un riesgo para el desarrollo psíquico del infante.

\section{Infant Facial Expressions of Emotions from Looking at Pictures (IFEEL)}

La prueba IFEEL (Emde, Osofsky \& Butterfield, 1993), fue creada con el objetivo de explorar e identificar los significados que una persona otorga a las señales afectivas que se expresan en el rostro de niños pequeños (Butterfield, Emde \& Osofsky, 1987; Meurs \& Vliegen, 2009). Una serie de fotografías, en las cuales se visualizan niños expresando diferentes emociones, funcionan como estímulos que alientan la expresión de las emociones que los cuidadores consideran que los nińos sienten, mostrando así la comprensión que tienen acerca del lenguaje emocional de estos (Hiltunen et al.,1999). Adicionalmente, el IFEEL permite estudiar diferencias individuales y grupales en la interpretación de las emociones de infantes, tanto en ámbitos clínicos como no clínicos, proporcionando una estructura general para entender los diferentes fenómenos que pueden surgir cuando se trata de interpretar las emociones de los infantes (Meurs \& Vliegen, 2009).

Emde y sus colaboradores (1993) se centraron en los significados otorgados a expresiones faciales más imprecisas de las emociones, reconociendo que existe una gran variabilidad en la interpretación de los afectos. Por tal motivo, la mayoría de las fotos del IFEEL (Emde, Osofsky \& Butterfield, 1993) presenta afectos de una intensidad moderada o imprecisa, y además, el fondo de estas — que podría brindar indicios situacionales de las expresiones afectivas - es neutral, de modo que el sujeto evaluado tiene la oportunidad de construir significados 
a partir de su interpretación en las respuestas. Por este motivo, el IFEEL (Emde, Osofsky \& Butterfield, 1993) podría ser considerado también como una prueba proyectiva, permitiendo profundizar en el mundo emocional de los cuidadores (Osofsky, Drell, \& Hann, 1993; Sazjnberg, 1993).

Adicionalmente, la utilidad del IFEEL (Emde, Osofsky \& Butterfield, 1993) reside en que hace posible construir un puente entre el ámbito experimental y la psicopatología del desarrollo psicodinámica. Por ejemplo, la investigación con niños de diferentes grupos de edad, desarrollada tanto en poblaciones normales como clínicas, hace posible tener una perspectiva psicopatológica del desarrollo. Es decir, la prueba permite determinar si el desarrollo se está dando de forma saludable, a través de la presencia de la amplitud del rango de emociones, así como una mayor presencia de emociones negativas y/o retraimiento (Emde, 1993). Por otro lado, el IFEEL permite detectar a las madres que sistemáticamente interpretan las señales afectivas de los bebés de forma inadecuada, permitiendo intervenir a tiempo en la diada, antes de que el niño interiorice la comunicación afectiva distorsionada, afectando su desarrollo afectivo (Meurs \& Vliegen, 2009).

En síntesis, el IFEEL es un instrumento que permite analizar la capacidad de un individuo para la lectura de las emociones, y además, determinar si existe algún grado de distorsión en esta lectura, por ejemplo, al perseverar en cierta respuesta o al brindar respuestas que se alejan del grupo normativo. Asimismo, el IFEEL permite conocer la amplitud del repertorio emocional de un cuidador (a través de la variedad de respuestas brindadas), así como su grado de modulación emocional (a través de la intensidad en la forma de verbalizar las emociones). Finalmente, a través de este instrumento, se puede detectar si existe un predominio de emociones negativas (presencia de una alta frecuencia de las emociones negativas: tristeza, cautela/timidez/reserva, vergüenza/ culpa, disgusto/desagrado, cólera, sufrimiento/angustia, y miedo), o si existe una confusión en la lectura emocional (presencia de un perfil emocional bimodal con grupos de emociones opuestos). 
Debido a la gran utilidad de este instrumento, este ha sido utilizado en diversas investigaciones con una gran variedad de poblaciones en riesgo, buscando detectar la influencia que ciertas variables contextuales y culturales pueden ejercer en la interacción entre cuidadores y bebés, las cuales pueden tener efectos de gran alcance y larga duración en su desarrollo. Así, Hiltunen, Moilanen, Szanjberg y Gardner (1999)utilizaron la prueba para comparar las respuestas emocionales de las madres antes de la guerra de Croacia con madres de la post guerra, con la finalidad de observar la incidencia de este hecho en la calidad de sus respuestas emocionales. En esta misma línea, Knezevic \& Jovancevic (2004) usaron el IFEEL para investigar el modo en que un grupo de madres croatas que pasaron experiencias traumáticas durante la guerra, perciben e interpretan las expresiones faciales de sus nińos. En ambos casos, se obtuvieron resultados significativos que daban cuenta de la nociva influencia que ejercía el contexto de guerra en la capacidad de estas madres para responder emocionalmente a las señales de sus bebés.

Con respecto a las propiedades psicométricas de la prueba original, Emde y sus colaboradores han realizado diversos estudios para comprobar su validez. Así, encontramos que el primer estudio de validez (Emde, Gaensbauer \& Harmon, 1976 citados en Meurs \& Vliegen, 2009) examinaba si las expresiones faciales de las fotos provocaban la producción de palabras emocionales, comprobando que más del 95\% de las palabras emitidas en respuesta a las fotos eran, en efecto, expresiones emocionales. Por lo tanto, Emde (citado en Meurs \& Vliegen, 2009) concluyó que las expresiones faciales aparentemente universales actúan como un canal para la comunicación afectiva.

Por otro lado, Emde (citado en Meurs \& Vliegen, 2009) buscó determinar si la prueba mide percepción o interpretación de emociones. Así, comprobó que mientras más imprecisa es la emoción retratada en una foto, más pequeño es el porcentaje de respuestas que esta recibe en la categoría afectiva más dominante. Si bien para cada foto existe una categoría afectiva dominante, también existe además una suficiente variabilidad en las posibles respuestas, conocida como "rango normal 
de variabilidad en la interpretación”. Algunos autores piensan que este "rango" es el reflejo de la proyección de los propios patrones de interpretación de afecto (Meurs \& Vliegen, 2009).

Otro estudio de validez realizado por Osofsky y Culp (1993) es aquel que analiza la percepción de emociones en madres adolescentes, encontrando que estas madres muestran un mayor número de respuestas de tristeza y alegría en comparación con un grupo de adolescentes no madres y con un grupo de madres de mayor edad, demostrando así la ambivalencia característica de la maternidad en edades tempranas. De este modo, se demuestra que el IFEEL es un instrumento que detecta a grupos que se encuentren en riesgo.

Finalmente, un estudio realizado con madres en riesgo de cometer maltrato infantil (Butterfield, 1993) encontró que estas madres tienden a nombrar una menor variedad de emociones al evaluar las fotos, y además, muestran emociones más extremas e intensas que aquellas madres que no se encuentran en riesgo de cometer maltrato. Adicionalmente, el IFEEL demuestra que estas madres brindan mayores respuestas de vergüenza junto con percepciones inapropiadas de alegría extrema, además de bajas respuestas de interés, timidez, miedo y tensión. Con este estudio, se sugiere que el IFEEL tiene una capacidad potencial para detectar y describir clínicamente ciertos grupos de madres y padres en riesgo.

\section{Método}

\section{Participantes}

La muestra fue seleccionada de manera intencional dado que las personas de NSE D y E fueron escogidas entre quienes asistían a un centro de estimulación infantil mientras que las de NSE B y C a través de la técnica "bola de nieve".

En el presente estudio participaron 363 personas de nivel socioeconómico (NSE) B y C (Medio: 53\%) y D y E (Bajo: 47\%), categorizados 
según la referencia de IPSOS-Apoyo (2009). El rango de edad de los participantes fue de 19 a 45 años de edad ( $M=26.63$ y $D E=5.69)$, en su mayoría mujeres jóvenes (64.5\%), entre 19 y 25 años (55.3\%), casados o convivientes (40\%) y procedentes de la ciudad de Lima (79.8\%). Aproximadamente un tercio de los participantes aún no tuvo hijos y otro tercio de ellos ha tuvo únicamente un hijo.

En todos los casos se contó con el consentimiento informado de los participantes previo a la administración de la prueba y se salvaguardó la confidencialidad de la identidad y datos de los participantes a lo largo del estudio.

\section{Medición}

El IFEEL Versión Peruana consta de 25 fotografías de niños con rasgos étnicos peruanos. La consigna de la prueba es: Aqui tenemos algunas fotos de expresiones faciales de niños. Díganos, en una palabra de ser posible, la emoción más fuerte y clara que cada niño está expresando en la foto. No hay respuestas buenas o malas, por favor responda con lo primero que se le venga a la mente.

Además, la prueba admite la posibilidad de brindar una ayuda si es que la persona parece no haber entendido las instrucciones iniciales o si muestra dificultad para nombrar alguna emoción: Te voy a dar una lista de palabras que pueden ser; estas palabras son interés, alegría, tristeza, miedo, sorpresa, disgusto, vergüenza, satisfacción, pasividad, precaución, cólera, sufrimiento, picardía y engreimiento.

Asimismo, se debe considerar que en la versión peruana de la prueba, la primera fotografía funciona como ejemplo, facilitando la introducción de la tarea y la comprensión de la consigna por lo que las respuestas dadas a este estímulo no son calificadas ni interpretadas.

Las respuestas libres brindadas a las demás fotografías son categorizadas utilizando el léxico de emociones, construido a partir de las respuestas dadas por la muestra de estudio (Anexo A). Estas categorías son catorce: Sorpresa, Interés, Alegría, Satisfacción, Pasividad, Tristeza, Cautela/timidez/reserva, Vergüenza/culpa, Disgusto/desagrado, 
Cólera, Sufrimiento/angustia, Miedo, Picardía y Engreimiento. Cabe señalar que las dos últimas se añadieron al léxico de la versión original considerando que reflejan particularidades de la población peruana. Adicionalmente, existe una categoría de respuestas de Emociones mezcladas que se refiere a aquellas respuestas en las que en una sola palabra se expresan distintas emociones. Asimismo, existen ciertas respuestas que al no expresar ninguna emoción no pueden ser calificadas y que se denominan No codificables, tales como intimidad, distracción, gritando, repitiendo, respondiendo entre otras.

La frecuencia de respuestas de cada uno de los grupos de emoción es interpretada de acuerdo a los valores referentes brindados en este artículo. Cabe destacar que adicionalmente a la frecuencia por grupo de emoción, se obtiene el número de respuestas de emociones agradables (suma de las respuestas de los grupos de sorpresa, interés, alegría y satisfacción) y emociones desagradables (suma de las respuestas de los grupos de pasividad, tristeza, cautela/timidez/reserva, vergüenza/ culpa, disgusto/desagrado, cólera, sufrimiento/angustia y miedo). De la misma manera, estos valores son comparados con los valores referenciales pertinentes.

\section{Procedimiento}

Para la creación de esta versión, se recolectaron fotografías de niños con rasgos faciales propios de la población peruana. Para este propósito, se obtuvo una muestra aproximadamente 450 fotografías de ocho niños y niñas de diferentes rasgos físicos. Luego se realizó un proceso de selección en diversas etapas:

- Primero se descartaron fotografías por su falta de nitidez, ángulo del rostro mayor a $45^{\circ}$, presencia de otros objetos en el fondo de la fotografía o su duplicidad. En este momento se seleccionaron 123 fotografías.

- Luego, las fotografías seleccionadas fueron evaluadas por el equipo de investigación conformado por cuatro psicólogas, quienes buscaron determinar el grado de ambigüedad de la expresión emocional 
en cada foto. De este proceso quedaron 52 fotografías con un grado de ambigüedad media de 3.29 (en una escala del 1 al 5) y DE de 1.32 .

- Seguidamente, estas 52 fotografías fueron administradas a un grupo de 154 estudiantes de psicología de una universidad de Lima con el objetivo de asegurar una suficiente variedad de las expresiones emocionales para la prueba en su conjunto. Sobre la base de la frecuencia de las respuestas dadas por los participantes, se eligió un grupo de 35 fotos que reproducía los diferentes grupos de emociones considerados en el léxico original de la prueba.

La administración de esta versión inicial del IFEEL de 35 fotografías se realizó en dos etapas, la primera a un grupo de 190 madres que acudían a un centro de estimulación de NSE bajo de la ciudad de Lima. Con las respuestas obtenidas, se procedió a crear un léxico propio, en el cual se incluyeron todas las formas de verbalización para cada uno de los grupos de emociones. Adicionalmente, a partir de las respuestas, se crearon las dos nuevas categorías de emociones anteriormente descritas. Una segunda etapa fue la administración de la prueba a un grupo de 173 hombres y mujeres, padres y no padres de NSE medio de la ciudad de Lima.

A partir de las respuestas de los participantes de ambos NSEs, se seleccionaron aquellas fotografías en las que menos del 70\% de participantes dieron como respuesta una única emoción. Se buscó además el balance en la frecuencia de los grupos de emociones en la prueba en su conjunto. De esta manera, la versión final del IFEEL Versión Peruana está compuesta por 25 fotos.

La confiabilidad test-retest fue calculada utilizando la prueba $T$ de Wilcoxon, así como el coeficiente de correlación de Spearman, tanto para cada grupo de emoción como para el total de respuestas. Para los análisis estadísticos se utilizaron estadísticos descriptivos y pruebas no paramétricas debido a que la distribución de frecuencias en los grupos no es normal. 


\section{Resultados}

A continuación se presentan los resultados del estudio, empezando por los resultados del análisis de confiabilidad. En segundo lugar, se presentan las comparaciones de las frecuencias en cada grupo de emoción en base al NSE, cabe destacar que esta variable es la que mostró diferenciar más las respuestas de los participantes por lo que no se incluyen los análisis realizados de acuerdo al género, edad y parentalidad de los participantes. Finalmente, se presenta el perfil de interpretación de la prueba.

Para el cálculo de la confiabilidad test-retest, se trabajó con un grupo de 50 personas, quienes fueron evaluadas dos veces en un lapso de 3 meses. Tal como se muestra en la Tabla 1, los únicos grupos que no tienen una adecuada confiabilidad son el de Verguenza/culpa y el de Emociones mezcladas. Por otro lado, se determinó que el coeficiente de correlación para el total de las respuestas es de 0.7 , indicando una buena correlación entre las puntuaciones obtenidas en ambas mediciones.

\section{Tabla 1}

Coeficientes de confiabilidad para los grupos de emoción

\begin{tabular}{llll}
\hline Grupo de emoción & $r$ & Grupo de emoción & $r$ \\
\hline Sorpresa & $0.57^{*}$ & Disgusto/Desagrado & $0.71^{*}$ \\
Interés & $0.61^{*}$ & Cólera & $0.48^{*}$ \\
Alegría & $0.75^{*}$ & Sufrimiento/Angustia & $0.64^{*}$ \\
Satisfacción & $0.39^{*}$ & Miedo & $0.60^{*}$ \\
Pasividad & $0.67^{*}$ & Picardía & $0.49^{*}$ \\
Tristeza & $0.78^{*}$ & Engreimiento & $0.39^{*}$ \\
Cautela/Timidez/Reserva & $0.61^{*}$ & Emociones mezcladas & 0.06 \\
Verguenza/Culpa & -0.04 & & \\
\hline${ }^{*} p<.05$ & & &
\end{tabular}


La Tabla 2 muestra que 10 de los 14 grupos de emociones muestran diferencias en su frecuencia de acuerdo al NSE del evaluado. Los NSEs D y E tienen mayor cantidad de respuestas en los grupos de emociones Alegría, Tristeza y Cólera mientras que los NSE B y C puntúan más alto en Interés, Satisfacción, Cautela/Timidez/Reserva, Vergüenza/ Culpa, Disgusto/Desagrado y Engreimiento.

\section{Tabla 2}

Comparación de las frecuencias por grupo de emoción en base al NSE

\begin{tabular}{|c|c|c|c|c|c|c|}
\hline \multirow{2}{*}{ Grupos de emoción } & \multicolumn{2}{|c|}{ NSE B-C } & \multicolumn{2}{|c|}{ NSE D-E } & \multirow{2}{*}{$U$} & \multirow{2}{*}{$Z$} \\
\hline & M & DS & M & DS & & \\
\hline Sorpresa & 1.05 & 1.28 & 1.25 & 1.83 & 16067.5 & -0.37 \\
\hline Interés & 2.39 & 2.25 & 1.80 & 2.23 & 13434.5 & $-3.07^{*}$ \\
\hline Alegría & 1.31 & 1.09 & 2.26 & 1.59 & 10292.5 & $-6.34^{*}$ \\
\hline Satisfacción & 1.59 & 1.91 & 1.32 & 1.94 & 14068.5 & $-2.48^{*}$ \\
\hline Pasividad & 2.73 & 2.50 & 3.02 & 2.76 & 15700.5 & -0.73 \\
\hline Tristeza & 3.28 & 2.35 & 4.96 & 3.39 & 11322 & $-5.15^{*}$ \\
\hline Cautela/Timidez/Reserva & 1.66 & 1.79 & 0.44 & 0.89 & 9248 & $-7.85^{*}$ \\
\hline Vergüenza/Culpa & 0.23 & 0.69 & 0.05 & 0.35 & 14505 & $-3.742^{*}$ \\
\hline Disgusto/Desagrado & 2.94 & 2.24 & 1.33 & 1.62 & 9010 & $-7.56^{*}$ \\
\hline Cólera & 1.42 & 1.77 & 2.76 & 2.33 & 10181 & $-6.38^{*}$ \\
\hline Sufrimiento/Angustia & 1.26 & 1.63 & 1.10 & 1.68 & 15228 & -1.28 \\
\hline Miedo & 2.61 & 2.54 & 2.82 & 2.99 & 16375 & -0.04 \\
\hline Picardía & 0.05 & 0.24 & 0.04 & 0.21 & 16213.5 & -0.63 \\
\hline Engreimiento & 0.75 & 1.31 & 0.12 & 0.39 & 11846 & $-6.09^{*}$ \\
\hline Emociones mezcladas & 0.30 & 0.66 & 0.14 & 0.41 & 14723 & $-2.59^{*}$ \\
\hline
\end{tabular}

${ }^{*} p<.05$ 
Por otro lado, se agruparon los grupos de emociones en dos grandes agrupaciones: emociones agradables y desagradables. Dado que se siguieron los procedimientos utilizados en la prueba original, no se consideraron en la agrupación los dos nuevos grupos de emociones: Picardía y Engreimiento. En todos los niveles socioeconómicos las emociones desagradables son significativamente mayores a las emociones agradables $(Z$ de -11.68 y -10.97 significativo a $p<.05$ para el NSE B-C y D-E respectivamente).

Adicionalmente se compararon las respuestas agradables y desagradables entre los NSEs, los resultados de estos análisis mostrados en la Tabla 3 nos indican que no existen diferencias en las frecuencias de las emociones negativas y las positivas entre los dos NSE.

\section{Tabla 3}

Comparación de las frecuencias para emociones agradables y desagradables en base al NSE

\begin{tabular}{lccccc}
\hline \multirow{2}{*}{ Grupo de emoción } & \multicolumn{2}{c}{ NSE B-C } & \multicolumn{2}{c}{ NSE D-E } & \multirow{2}{*}{$Z$} \\
\cline { 2 - 5 } & \multicolumn{1}{c}{ M } & DS & M & DS & \\
\hline Agradables & 6.33 & 2.73 & 6.63 & 3.08 & -1.05 \\
Desagradables & 16.15 & 3.28 & 16.49 & 3.36 & -0.76 \\
\hline${ }^{*} p<.05$ & & & & &
\end{tabular}

Finalmente, en las Tablas $4 \mathrm{a}$ y $4 \mathrm{~b}$ se presentan los perfiles de interpretación de los valores de las frecuencias de respuestas para cada grupo de emoción y para las respuestas agradables y desagradables por NSE. 


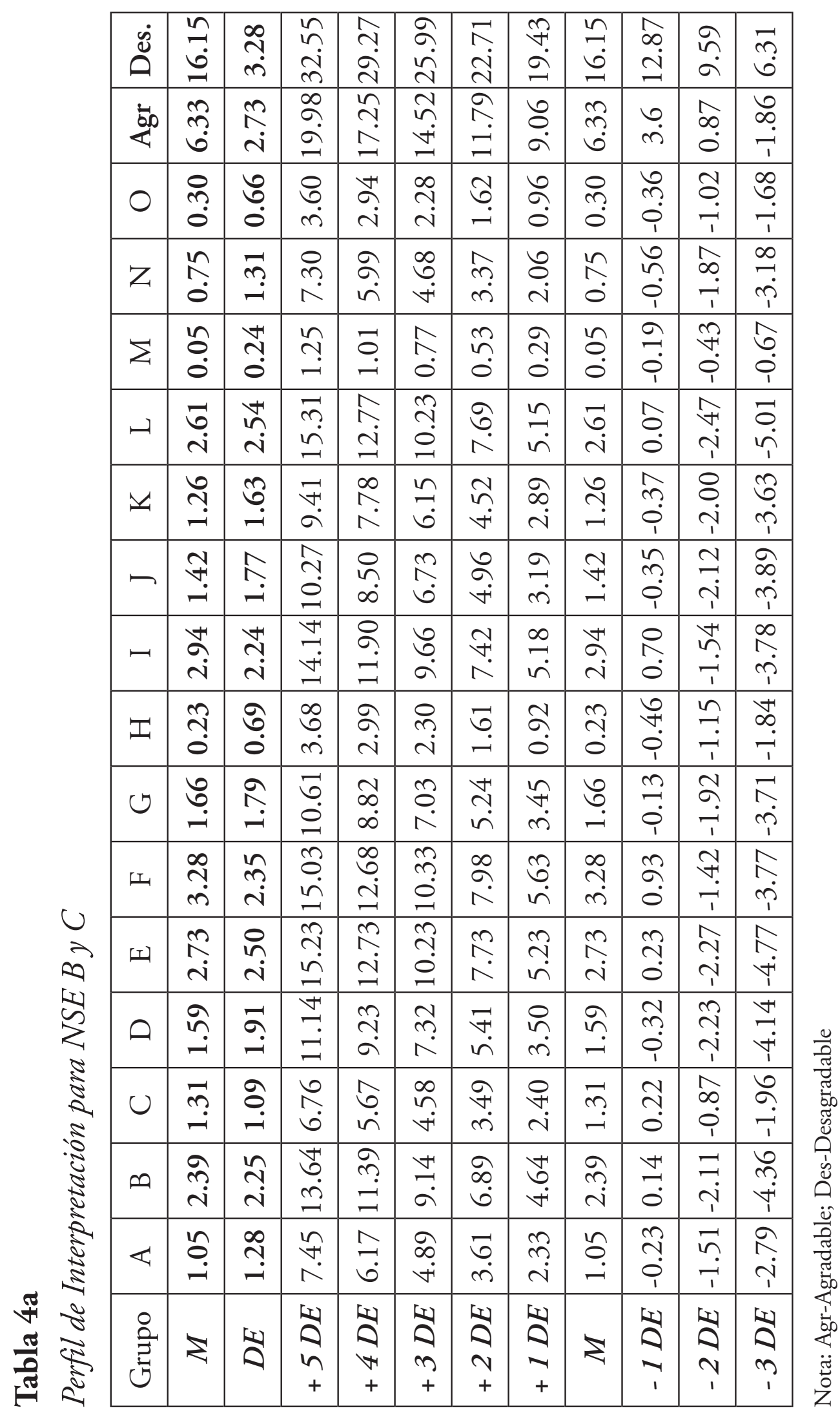




\begin{tabular}{|c|c|c|c|c|c|c|c|c|c|c|c|c|}
\hline & $\stackrel{\dot{\Delta}}{\stackrel{\Delta}{0}}$ & $\begin{array}{l}\stackrel{\vartheta}{\mathrm{f}} \\
\text { }\end{array}$ & $\begin{array}{l}\stackrel{\leftrightarrow}{n} \\
\stackrel{n}{n}\end{array}$ & $\begin{array}{l}\overrightarrow{\hat{y}} \\
\dot{m} \\
\tilde{\infty}\end{array}$ & $\begin{array}{l}\tilde{\hat{\lambda}} \\
\stackrel{\hat{i}}{2}\end{array}$ & $\begin{array}{l}\hat{n} \\
\hat{\sigma}\end{array}$ & $\begin{array}{l}\overrightarrow{\vec{\sim}} \\
\tilde{\sim}\end{array}$ & 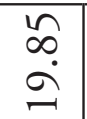 & $\begin{array}{l}\vec{\vartheta} \\
\text { }\end{array}$ & $\begin{array}{l}m \\
\vec{n}\end{array}$ & $\stackrel{\hat{\alpha}}{\hat{\alpha}}$ & $\vec{F}$ \\
\hline & $\underset{0}{4}$ & గ్రి & $\begin{array}{l}\infty \\
\dot{n} \\
\dot{n}\end{array}$ & $\begin{array}{l}m \\
\text { in } \\
\tilde{N}\end{array}$ & $\begin{array}{l}\stackrel{n}{\infty} \\
\infty \\
\sim\end{array}$ & $\begin{array}{l}\hat{\infty} \\
\hat{n}\end{array}$ & 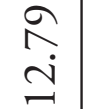 & $\stackrel{\vec{a}}{a}$ & రి & $\begin{array}{l}\tilde{n} \\
\dot{n}\end{array}$ & $\underset{\sim}{\stackrel{f}{0}}$ & $\begin{array}{l}\vec{\sigma} \\
\underset{i}{i}\end{array}$ \\
\hline & 0 & $\stackrel{t}{0}$ & $\underset{0}{F}$ & $\overrightarrow{\vec{i}}$ & 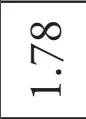 & $\stackrel{\hat{n}}{\rightarrow}$ & 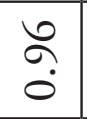 & $\begin{array}{l}\hat{n} \\
\tilde{o}\end{array}$ & $\begin{array}{l}\stackrel{+1}{*} \\
\stackrel{0}{0}\end{array}$ & $\begin{array}{l}\hat{y} \\
\hat{i}\end{array}$ & \begin{tabular}{|l|}
0 \\
0 \\
0 \\
0
\end{tabular} & oे \\
\hline & z & $\stackrel{7}{7}$ & ஸे & $\hat{\stackrel{\hat{i}}{ }}$ & : & 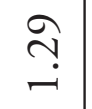 & $\stackrel{̊}{\circ}$ & $\overrightarrow{\tilde{n}}$ & $\stackrel{T}{\stackrel{2}{0}}$ & $\begin{array}{l}\hat{y} \\
\vdots \\
i\end{array}$ & $\begin{array}{l}0 \\
0 \\
0 \\
0\end{array}$ & $\begin{array}{l}n \\
\hat{r} \\
i\end{array}$ \\
\hline & $\Sigma$ & 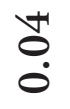 & $\overrightarrow{\tilde{O}}$ & $\stackrel{\partial}{\text { Oे }}$ & $\begin{array}{l}\infty \\
\infty \\
0 \\
0\end{array}$ & $\begin{array}{l}\hat{0} \\
0\end{array}$ & $\begin{array}{l}\stackrel{0}{+} \\
0\end{array}$ & 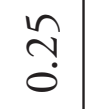 & $\begin{array}{l}\stackrel{+}{0} \\
\stackrel{0}{0}\end{array}$ & $\begin{array}{l}\overrightarrow{0} \\
\vec{i}\end{array}$ & $\mid \begin{array}{l}\infty \\
n \\
0 \\
1\end{array}$ & $\begin{array}{l}\hat{\tilde{n}} \\
\hat{\varphi}\end{array}$ \\
\hline & - & $\begin{array}{l}\underset{\infty}{\infty} \\
\text { i }\end{array}$ & $\hat{\widehat{i}}$ & $\stackrel{尺}{\stackrel{1}{\Im}}$ & $\underset{\underset{+}{\infty}}{\stackrel{\infty}{+}}$ & $\begin{array}{l}\stackrel{શ}{I} \\
=\end{array}$ & $\begin{array}{l}\infty \\
\infty \\
\infty\end{array}$ & $\begin{array}{l}\vec{\sigma} \\
\dot{n} \\
\dot{n}\end{array}$ & 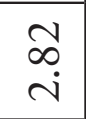 & $\stackrel{\overrightarrow{1}}{\overrightarrow{0}}$ & $\begin{array}{l}0 \\
\dot{p} \\
\vec{r}\end{array}$ & $\begin{array}{l}n \\
\stackrel{n}{n} \\
1\end{array}$ \\
\hline & $\simeq$ & $\stackrel{\overbrace{}}{\longrightarrow}$ & 象 & $\begin{array}{c}\stackrel{n}{2} \\
\alpha\end{array}$ & $\underset{\sim}{\stackrel{N}{\sim}}$ & $\begin{array}{l} \pm \\
\vec{\sigma}\end{array}$ & 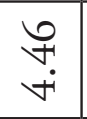 & 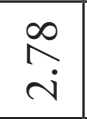 & $\stackrel{\leftrightarrow}{\rightleftarrows}$ & 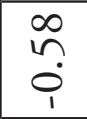 & \begin{tabular}{l}
0 \\
\multirow{1}{*}{} \\
$i$ \\
$i$
\end{tabular} & $\begin{array}{l}\vec{t} \\
\vec{r}\end{array}$ \\
\hline & $\sim$ & 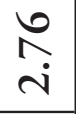 & $\stackrel{n}{i}$ & \begin{tabular}{|l}
$\vec{F}$ \\
$\dot{I}$
\end{tabular} & 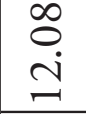 & $\begin{array}{l}\mathfrak{n} \\
\hat{a}\end{array}$ & $\underset{t}{\stackrel{T}{\sim}}$ & $\begin{array}{l}\hat{o} \\
\dot{r}\end{array}$ & $\begin{array}{l}\stackrel{゚}{i} \\
i\end{array}$ & $\stackrel{\leftrightarrow}{\stackrel{\leftrightarrow}{0}}$ & 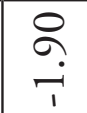 & 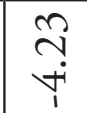 \\
\hline & - & $\stackrel{m}{\rightarrow}$ & $\underset{\sim}{\tilde{C}}$ & $\stackrel{\stackrel{m}{+}}{\stackrel{\sim}{\sim}}$ & $\stackrel{\infty}{\wedge}$ & $\vec{\sigma}$ & $\begin{array}{l}\hat{n} \\
\dot{+}\end{array}$ & $\begin{array}{l}\tilde{\hat{i}} \\
\hat{i}\end{array}$ & $\stackrel{m}{=}$ & $\begin{array}{l}\text { ָे } \\
\text { }\end{array}$ & $\vec{\sigma}$ & $\begin{array}{l}n \\
n \\
\tilde{r}\end{array}$ \\
\hline & $I$ & $\stackrel{n}{o}$ & ñ & $\stackrel{\infty}{\infty}$ & $\stackrel{n}{\stackrel{n}{-}}$ & $\begin{array}{l}\stackrel{0}{\leftrightarrows} \\
ت\end{array}$ & $\begin{array}{l}\mathfrak{n} \\
\hat{0}\end{array}$ & $\begin{array}{l}\stackrel{+}{0} \\
0\end{array}$ & $\begin{array}{l}n \\
0 \\
\dot{0}\end{array}$ & $\begin{array}{l}0 \\
n ! \\
i\end{array}$ & \begin{tabular}{|l}
$n$ \\
6 \\
0 \\
1
\end{tabular} & \begin{tabular}{|l|}
8 \\
0 \\
\end{tabular} \\
\hline & ט & $\stackrel{\Downarrow}{\sharp}$ & $\begin{array}{l}\infty \\
0 \\
0\end{array}$ & $\mid \begin{array}{l}a \\
\infty \\
\forall+\end{array}$ & $\underset{+}{\stackrel{\&}{+}}$ & $\overrightarrow{\vec{n}}$ & 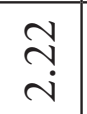 & $\stackrel{m}{\rightarrow}$ & 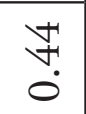 & $\begin{array}{l}\text { fof. } \\
\text { po }\end{array}$ & 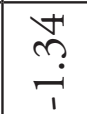 & $\begin{array}{l}\tilde{n} \\
i \\
i\end{array}$ \\
\hline & 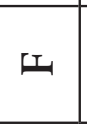 & $\underset{\forall}{\stackrel{v}{~}}$ & $\begin{array}{l}\hat{m} \\
\dot{n}\end{array}$ & $\begin{array}{l}\overrightarrow{\hat{\alpha}} \\
\vec{\lambda}\end{array}$ & $\begin{array}{l}\tilde{N} \\
\underset{\infty}{\sim}\end{array}$ & $\begin{array}{l}m \\
\vec{n}\end{array}$ & $\begin{array}{l}\stackrel{+}{\Xi} \\
=\end{array}$ & $\begin{array}{l}\tilde{n} \\
\infty \\
\infty\end{array}$ & 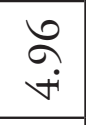 & $\stackrel{n}{n}$ & $\begin{array}{c}2 \\
0 \\
-1 \\
\end{array}$ & $\begin{array}{l}\overrightarrow{\tilde{n}} \\
\tilde{r}\end{array}$ \\
\hline & 피 & $\tilde{\tilde{r}}$ & $\begin{array}{l}\stackrel{2}{i} \\
\text { in }\end{array}$ & 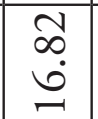 & $\begin{array}{l}\stackrel{\circlearrowright}{ } \\
\stackrel{+}{+}\end{array}$ & $\begin{array}{l}\stackrel{\oplus}{=} \\
=\end{array}$ & \begin{tabular}{l}
$\stackrel{+}{\sim}$ \\
\multirow{\infty}{*}{}
\end{tabular} & $\begin{array}{l}\infty \\
\stackrel{\infty}{n} \\
\end{array}$ & $\begin{array}{l}\tilde{\sigma} \\
\dot{n}\end{array}$ & 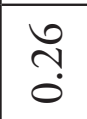 & $\begin{array}{l}0 \\
\stackrel{n}{n} \\
i\end{array}$ & $\begin{array}{l}\text { İ } \\
\text { nu }\end{array}$ \\
\hline & D & $\widetilde{\Omega}$ & $\stackrel{+}{S}$ & $\begin{array}{l}\tilde{S} \\
\dot{\Xi} \\
\end{array}$ & $\begin{array}{l}\infty \\
\stackrel{\infty}{\circ} \\
\stackrel{\circ}{0}\end{array}$ & $\begin{array}{l} \pm \\
\end{array}$ & $\begin{array}{l}\text { ì } \\
\text { in }\end{array}$ & 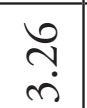 & $\stackrel{\sim}{\sim}$ & $\begin{array}{l}0 \\
0 \\
0\end{array}$ & $\begin{array}{l}0 \\
\stackrel{0}{n} \\
i\end{array}$ & $\begin{array}{l}\stackrel{i}{n} \\
i \\
\forall\end{array}$ \\
\hline & u & ্ָ & $\stackrel{\overbrace{}}{\tilde{\gamma}}$ & $\overrightarrow{\vec{r}}$ & $\begin{array}{l}\widetilde{W} \\
\infty\end{array}$ & $\stackrel{0}{0}$ & $\begin{array}{l}\sharp \\
\forall \\
\sim\end{array}$ & $\begin{array}{l}n \\
\infty \\
n\end{array}$ & $\begin{array}{l}\vec{U} \\
i\end{array}$ & $\underset{0}{0}$ & $\begin{array}{l}\alpha \\
\dot{\varphi} \\
\hat{\varphi}\end{array}$ & $\begin{array}{l}\overrightarrow{\widehat{\Upsilon}} \\
\hat{\imath}\end{array}$ \\
\hline & $\infty$ & 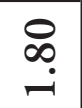 & $\stackrel{\sim}{\sim}$ & $\begin{array}{l}\stackrel{n}{\hat{I}} \\
\underline{i}\end{array}$ & 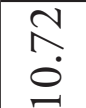 & $\begin{array}{l}\vec{f} \\
\infty \\
\infty\end{array}$ & $\begin{array}{l}\stackrel{\sim}{ } \\
\text { U. }\end{array}$ & $\begin{array}{c}\tilde{O} \\
\stackrel{\forall}{+}\end{array}$ & $\begin{array}{l}\infty \\
\stackrel{\infty}{-}\end{array}$ & $\begin{array}{l}\stackrel{f}{f} \\
\stackrel{p}{i}\end{array}$ & $\begin{array}{l}0 \\
0 \\
i \\
i\end{array}$ & $\begin{array}{l}\curvearrowright \\
\infty \\
\dot{1}\end{array}$ \\
\hline 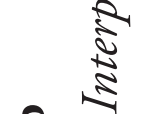 & $\ll$ & $\stackrel{\sim}{\stackrel{n}{\tau}}$ & $\stackrel{\infty}{\infty}$ & 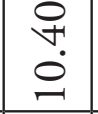 & 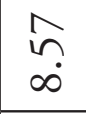 & $\stackrel{+}{\hat{\sigma}}$ & $\overrightarrow{\vec{\nabla}}$ & $\begin{array}{l}\infty \\
\dot{r} \\
\dot{r}\end{array}$ & $\stackrel{\overbrace{}}{\stackrel{n}{\vartheta}}$ & $\begin{array}{l}\infty \\
\dddot{0} \\
i\end{array}$ & 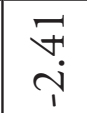 & $\underset{\sim}{\stackrel{\sharp}{+}}$ \\
\hline है & 总 & $\Sigma$ & 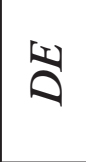 & $\begin{array}{l}w \\
\Delta \\
n \\
+\end{array}$ & $\begin{array}{l}\text { y } \\
\text { a } \\
+\end{array}$ & $\begin{array}{l}\begin{array}{l}1 \\
0 \\
o \\
+\end{array} \\
0\end{array}$ & 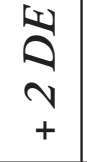 & $\begin{array}{l}y \\
a \\
+ \\
+\end{array}$ & $\Sigma$ & $\frac{u^{2}}{i}$ & \begin{tabular}{l}
1 \\
$a$ \\
\multirow{1}{1}{}
\end{tabular} & $\begin{array}{l}w \\
\text { n } \\
1\end{array}$ \\
\hline
\end{tabular}




\section{Discusión}

A continuación, discutiremos algunos puntos relevantes en la creación de la versión peruana del IFEEL, así como los resultados principales obtenidos a partir de la investigación.

Dadas las características físicas y culturales de la población peruana, se vio la necesidad de seguir una serie de pasos con el objetivo de obtener un instrumento para evaluar la particularidad de la interpretación de emociones en el contexto estudiado. Así, en primer lugar, las características étnicas de los niños de las fotografías ha sido uno de los elementos prioritarios en este estudio; luego de analizar los resultados, consideramos que estos facilitaron el proceso de identificación de los evaluados, permitiendo por tanto la obtención de una amplia gama de respuestas emocionales las cuales permitieron la creación de un amplio léxico de expresiones verbales que designan diferentes emociones.

Otro de los pasos centrales en esta investigación fue el proceso de selección de fotografías; la versión final de 25 fotografías fueron aquellas que desde los diversos procedimientos descritos, tenían un adecuado grado de ambigüedad para favorecer la producción de una mayor variedad de respuestas emocionales. Dados los matices particulares de cada una de las fotos seleccionadas y del conjunto de las 25 fotografías, el IFEEL Versión Peruana es una prueba diferente a la versión original. Sin embargo, dado que se reprodujeron los procedimientos seguidos por la prueba original, se puede considerar que es un método equivalente de evaluación de la interpretación de emociones al de Emde et al. (1993).

Se consideró necesario crear un léxico propio que muestre las particularidades de las verbalizaciones utilizadas para nombrar las emociones, de esta manera se cuenta con un referente de calificación adaptado al contexto. En primer lugar, se puede observar que estas verbalizaciones mayoritariamente reprodujeron los grupos de emoción de la prueba original lo cual apoya la idea de la universalidad de las emociones. Sin embargo y dada la elevada frecuencia de ciertas respuestas, 
se consideró incluir dos emociones adicionales, las de Engreimiento y Picardía. Si bien este aspecto necesita ser profundizado en posteriores estudios, cabe señalar que la emoción Engreimiento aparece con una connotación negativa indicando que en algunas ocasiones, las personas interpretan una alta demanda de afecto en la expresión de sus hijos. La Picardía, por otro lado, representa una característica propia de la cultura peruana, similar a la viveza y a la costumbre de sacar ventaja de diversas situaciones la cual también es atribuida a los niños.

Se encontró que el NSE es una variable que genera diferencias significativas en la manera como las personas interpretan emociones por lo que se optó por la creación de referentes de interpretación diferenciados en base a esta variable. Estos hallazgos estarían en relativa concordancia con el estudio de Hiltunen et al. (1998) que encontraron diferencias entre madres de diferentes culturas (americanas, croatas, finlandesas). Si bien el nivel socioeconómico y la cultura son variables diferentes, en Lima los sectores D y E están básicamente conformados por migrantes de primera y segunda generación de la sierra y selva, mientras que los sectores $\mathrm{B}$ y $\mathrm{C}$ son en su mayoría nacidos en Lima, lo cual podría hablar de diferencias culturales entre los NSE participantes en este estudio. Al respecto, podría pensarse que las diferentes condiciones de vida en el que las personas se desarrollan influye en su lectura de las expresiones emocionales. Es decir, la mayor presencia de emociones básicas en los sectores D y E, y la menor presencia de emociones complejas, podrían ser originadas por las diferencias en la capacidad verbal de ambos grupos. Es probable que los sectores $\mathrm{D}$ y $\mathrm{E}$ tengan más dificultades para expresar emociones complejas debido a que presentan niveles de vocabulario limitado, y no porque no experimenten las emociones. Más aún, es probable que estas emociones complejas se expresen a través de otros canales de comunicación no verbales. Esta hipótesis al igual que la menor presencia de diferencias en la interpretación de emociones por edad, género y parentalidad son aspectos que necesitan ser profundizados en estudios posteriores. 


\section{Referencias}

Ackerman, B. P. \& Izard, C. (2004). Emotion cognition in children and adolescents: Introduction to the special issue. Journal of Experimental Child Psychology, 84, 271-275.

Brazelton, T. B. \& Cramer, B. G. (1993). La relación más temprana. Padres, bebés y el drama del apego inicial. Barcelona: Paidós.

Butterfield, P. M. (1993). Responses to IFEEL Pictures in mothers at risk for child maltreatment. En R. N. Emde, J. D. Osofsky \& Butterfield, P. M. (Eds.), The IFEEL Pictures. A new instrument for interpreting emotions (pp.161-173). Madison, CT: International Universities Press, Inc.

Butterfield, P. M., Emde, R. N. \& Osofsky, J. D. (1987). IFEEL pictures manual. Infant facial expressions of emotion from looking at pictures. The Regents of the University of Colorado.

Emde, R. (1980). Emotional availability: A reciprocal reward system for infants and parents with implications for prevention of psychosocial disorders. En P. M. Taylor (Ed.), Parent-infant relationships (pp. 87-115). Orlando, FL: Grune \& Stratton.

Emde, R. N. (1993). A framework for viewing emotions. En R. N. Emde, J. D. Osofsky, \& P. M. Butterfield (Eds.), The IFEEL pictures. A new instrument for interpreting emotions (pp. 149-160). Madison, CT: International Universities Press, Inc.

Emde, R. N., Osofsky, J. D. \& Butterfield, P. M. (1993). The IFEEL Pictures: A new instrument for interpreting emotions. Madison: International Universities Press, Inc.

Fonagy, P., Gergely, G., Jurist, E. L. \& Target, M. (2002). Affect regulation, mentalization, and the development of the self. Nueva York: Other Press.

Fonagy, P. \& Target, M. (1997). Apego y la función reflexiva: su rol en la autorganización. Cambridge: Cambridge University Press.

Hiltunen, P., Moilanen, I., Szanjberg, N. \& Gardner, N. (1999). The IFEEL Pictures: Transcultural Aspects of Importing a New Method. Nordic Journal of Psychiatry, 53(3), 231-235. 
IPSOS-Apoyo Opinión y Mercado (2009). Niveles socioeconómicos de la gran Lima 2009. Lima: Apoyo.

Knezevic, M. \& Jovancevic, M. (2004). The IFEEL Pictures: Psychological Trauma and Perception, and Interpretation of Child's Emotions. Nordic Journal of Psychiatry 58 (2), 139 - 145.

Meurs, P. \& Vliegen, N. (2009). Affectinterpretatie en emotieregulatie: IFEEL Pictures Test (Nederlandstalige Versie) Manuscrito presentado para publicación.

Osofsky, J.D., Drell, M. \& Hann, D. (1993). IFEEL stories: pictures used as projective story stimuli. En R. N. Emde, J. D. Osofsky, \& P. M. Butterfield (Eds.), The IFEEL pictures. A new instrument for interpreting emotions (pp. 197-206). Madison, CT: International Universities Press, Inc.

Osofsky, J.D. \& Culp, A.M. (1993). Perceptions of infant emotions in adolescent mothers. En R. N. Emde, J. D. Osofsky, \& P. M. Butterfield (Eds.), The IFEEL pictures. A new instrument for interpreting emotions (pp. 3-26). Madison, CT: International Universities Press, Inc.

Sazjnberg, N.M. (1993). The IFEEL Pictures as a projective instrument: pre- and full-term mothers. En R. N. Emde, J. D. Osofsky, \& P. M. Butterfield (Eds.), The IFEEL pictures. A new instrument for interpreting emotions (pp. 207-216). Madison, CT: International Universities Press, Inc.

Stern, D. (1991). El mundo interpersonal del infante: Una perspectiva desde el psicoanálisis y la psicología evolutiva. Buenos Aires: Paidós.

Winnicott, D. W. (1962). Los designios del tratamiento psicoanalítico. En El proceso de maduración en el niño (p. 201). Barcelona: Editorial Laia.

Winnicott, D.W. (1982). Realidad y juego. Barcelona: Gedisa.

Zahn-Waxler, C. \& cols. (1993). Pictures of Infant Emotion: A Task for Assessing Mother's and Young Children's Verbal Communication about Affect. En R. N. Emde, J. D. Osofsky, \& P. M. Butterfield (Eds.), The IFEEL Pictures: A New Instrument for Interpreting Emotions (1ra. ed., pp.217-236). Madison, CT: International Universities Press. 


\section{Anexo A}

\section{Léxico de emociones}

\begin{tabular}{|l|l|}
\hline A. Sorpresa & $\begin{array}{l}\text { Asombro, impactado, impresionado, extrañado, } \\
\text { Admiración, deslumbrado }\end{array}$ \\
\hline B. Interés & $\begin{array}{l}\text { Mirando, observativo, contemplación, fascinado, } \\
\text { absorto, atraído, entretenido } \\
\text { Atención, concentración, ve algo que le llama la } \\
\text { atención } \\
\text { Expectación, alerta, intriga, curioso, curiosidad, } \\
\text { Expectativa, ganas, con ganas de, deseo, quiere algo, } \\
\text { espera, esperando, anhelo } \\
\text { Esperanza, ilusión }\end{array}$ \\
\hline C. Alegría & $\begin{array}{l}\text { Felicidad, gozo, regocijo, diversión, entusiasmo } \\
\text { Éxtasis, júbilo, euforia, exaltación, carcajada, } \\
\text { carcajeando, algarabía, optimismo } \\
\text { Sonrisa, contento, humor, risueño, emoción, placer } \\
\text { Amor, cariño, ternura, dulzura } \\
\text { Jugando, juguetón }\end{array}$ \\
\hline D. Satisfacción & $\begin{array}{l}\text { Tranquilidad, relajo, despreocupado, normal, frescura, } \\
\text { satisfacción, orgullo } \\
\text { Gusto, agrado, plenitud, gratificado } \\
\text { Paz, quietud, calma, sereno, paciente } \\
\text { Bien, comodidad, confianza, seguridad, confortable } \\
\text { Sueño, soñando } \\
\text { Libre }\end{array}$ \\
\hline E. Pasividad & $\begin{array}{l}\text { Sumisión } \\
\text { Resignación, conformidad } \\
\text { Desgano, flojera, aburrimiento, desinterés, indiferencia, } \\
\text { frialdad, apatía } \\
\text { Cansado, cansancio } \\
\text { Durmiendo, adormecido somnoliento } \\
\text { Reflexión, pensativo, seriedad, meditando, razonando }\end{array}$ \\
\hline
\end{tabular}




\begin{tabular}{|l|l|}
\hline F. Tristeza & $\begin{array}{l}\text { Pena, pesar, depresión, lástima } \\
\text { Infelicidad, no está feliz } \\
\text { Desánimo, decaído, apagado } \\
\text { Desilusión, decepción } \\
\text { Soledad, vacío, nostalgia, extrañando } \\
\text { Rechazado, relegado }\end{array}$ \\
\hline $\begin{array}{l}\text { G. Cautela / } \\
\text { timidez/ } \\
\text { reserva }\end{array}$ & $\begin{array}{l}\text { Desconfianza, sospecha, incredulidad, recelo, cautela, } \\
\text { precaución, suspicacia, calculador } \\
\text { Desorientación, confusión, desconcierto, aturdido } \\
\text { Incertidumbre, duda, incógnita, pregunta, interrogativa, } \\
\text { indecisión }\end{array}$ \\
$\begin{array}{l}\text { Incomprensión, desconocimiento, no sabe, } \\
\text { Extrañeza } \\
\text { Reprimido, cohibido, inhibido, inseguro, tímido } \\
\text { Misterioso }\end{array}$ \\
\hline $\begin{array}{l}\text { H. Vergüenza / } \\
\text { culpa }\end{array}$ & $\begin{array}{l}\text { Humillado, avergonzado, arrepentimiento } \\
\text { I. Disgusto / } \\
\text { desagrado }\end{array}$ \\
$\begin{array}{l}\text { Asco, reproche, rechazo, no le gusta, ve algo que no le } \\
\text { gusta, repudio } \\
\text { Molestia, fastidio, malestar, incomodidad, desazón } \\
\text { Disgusto } \\
\text { Malhumor, se siente mal } \\
\text { Disconforme, insatisfacción, inconformidad, } \\
\text { descontento, incontento, en desacuerdo, desacuerdo } \\
\text { Impaciencia } \\
\text { Tenso, presionado } \\
\text { Hambre, llanto }\end{array}$ \\
\hline $\begin{array}{l}\text { Rabia, enfado, reniego, enojo, irritado, furia, irritación, } \\
\text { ira, molesto, medio molesto, resondrando, alteración } \\
\text { Agresivo, peleando, prepotencia } \\
\text { Rencor, odio, envidia } \\
\text { Frustración n } \\
\text { Queja, oposición, reclamo, negativa, terco, rebeldía } \\
\text { Celos, picón } \\
\text { Resentida, resentimiento, indignación }\end{array}$ \\
\hline
\end{tabular}




\begin{tabular}{|c|c|}
\hline $\begin{array}{l}\text { K. Sufrimiento / } \\
\text { angustia }\end{array}$ & $\begin{array}{l}\text { Dolor, golpeado, abatimiento, inconsolado, destrozado } \\
\text { Desesperación } \\
\text { Impotencia, desesperanza } \\
\text { Angustia, acongojado, congoja, aflicción } \\
\text { Melancolía, desolación, desconsuelo, abandono } \\
\text { Amargura } \\
\text { Maltrato }\end{array}$ \\
\hline L. Miedo & $\begin{array}{l}\text { Susto, asustado, terror, aterrorizado, espantado, temor, } \\
\text { impresión } \\
\text { Nervioso, nerviosismo, ansiedad } \\
\text { Preocupación } \\
\text { Perturbado }\end{array}$ \\
\hline M. Picardía & $\begin{array}{l}\text { Burla, coqueto, bromista } \\
\text { Travieso }\end{array}$ \\
\hline N. Engreimiento & $\begin{array}{l}\text { Capricho, berrinche, mañoso, puchero } \\
\text { Fingiendo, disforzado }\end{array}$ \\
\hline $\begin{array}{l}\text { O. Emociones } \\
\text { mezcladas } \\
\text { (expresan una } \\
\text { emoción que } \\
\text { no puede ser } \\
\text { clasificada en las } \\
\text { anteriores o la } \\
\text { palabra puede } \\
\text { ser expresar } \\
\text { distintas } \\
\text { emociones) }\end{array}$ & $\begin{array}{l}\text { Firmeza, fortaleza, dureza, fuerza } \\
\text { Sensible, fragilidad } \\
\text { Ambivalencia } \\
\text { Insensibilidad } \\
\text { Consternación } \\
\text { Excitante } \\
\text { Inquietud, intranquilo } \\
\text { Rogar } \\
\text { Recordando } \\
\text { Niño necesitado, como si alguien le faltara, necesita cariño } \\
\text { Castigado, regañado }\end{array}$ \\
\hline
\end{tabular}

Recibido: 22 de febrero de 2012

Aceptado: 26 de julio de 2012 